\title{
Suppression of tunnel spalling by engineered rock mass damage
}

\author{
AC McDonald Queen's University, Canada \\ SD McKinnon Queen's University, Canada
}

\begin{abstract}
Near excavation boundaries in massive to moderately-jointed rock masses, rock predominately fails by extensional fracturing leading to the progressive formation of thin slabs of rock-referred to as spalls. These spalls can buckle, rotate, dilate and accumulate to cause bulking of the rock mass. Spalling can lead to overbreak, ground support damage and capacity consumption by bulking, and ejection of rock (strainbursting). Reliable design of deep excavations depends on reliable forecasts, which require informed understanding of the mechanisms of brittle failure and behaviour.

Confinement has been used as a strategy to suppress spalling but this is not always possible. Alternatively, spalling can be suppressed by the accumulation of damage (whether engineered or naturally occurring) to the rock mass. Through an analogue model of a brittle rock mass, we show that engineering damage into the rock mass supresses and can theoretically prevent the occurrence of spalling around an underground excavation. Furthermore, we show that the bimodular ratio, related to the modulus of the intact rock in compressional versus extensional loading, is an important indicator of damage and proneness to strainbursting.
\end{abstract}

Keywords: spalling, strainbursting, elasticity, engineered damage, brittle rock

\section{Introduction}

Spalling describes when small rock fragments on the immediate boundary of an excavation fail and are ejected into the opening. Spalling poses a hazard to mine personnel and production and causes additional costs to operations. As the depth of mining increases and extraction ratios increase, the potential for unstable conditions increases, as does the magnitude of risk and the need for tunnel development under high stress conditions.

Mining ground control has relied heavily on experience. This has led to the ubiquitous use of the Hoek-Brown failure criterion (Hoek et al. 2002) and rock mass classification methods such as Rock Mass Rating (RMR) (Bieniawski 1988) and Q (Norwegian Geotechnical Institute (NGI) 2015). The experience that led to these empirical methods is dominated by shallow conditions, leading to a bias in the interpretation of behaviour. When working in deep high stress conditions, or very high extraction ratios, methods based on current experience are not reliable and the relevant experience is insufficient to develop rigorous empirical methods. Mechanics-based analysis, when backed up by experimental and numerical data, can provide more reliable guidance for understanding brittle failure in these conditions.

Recent research by McDonald (2019) identified crack closure non-linear elasticity as a key factor in brittle extensional crack propagation. This provides a means for the identification of rock masses that have a higher likelihood for spalling and strainbursting. It also suggests that mitigation of higher hazard conditions can be achieved by creating this non-linearity through engineered rock mass damage. This paper applies the general crack propagation results of that research to the particular case of spalling around a deep excavation. 


\section{$2 \quad$ Background}

\subsection{Buckling}

One hypothesis for spalling is that thin fragments form, or may exist through geological conditions, close to the excavation boundary and buckle (Fairhurst \& Cook 1966). Buckling occurs when the beam end stress exceeds a critical limit. The limit depends on the slenderness of the fragment, so as cracks grow in the sidewall of an excavation, the slenderness increases, and the buckling limit can drop sufficiently to reach the applied stress. The critical buckling stress in Equation 1 was defined for rock beams by Fairhurst \& Cook (1966). This assumes a freely rotating beam end boundary condition.

$$
\text { Buckling Stress }=\frac{\pi^{2} E}{12\left(\frac{l}{t}\right)^{2}}
$$

where:

$$
\begin{array}{ll}
E & =\text { Young's modulus. } \\
I & =\text { beam length. } \\
t & =\text { beam thickness. }
\end{array}
$$

The deflection of the beam is also used to evaluate the effect of eccentric loading. Finally, the potential for beam crushing and beam snap-through can be assessed by comparing the induced stress concentrations within the beam to the compressive and tensile strength of the rock. These are the main potential failure mechanisms.

The slenderness of the beam depends on the propagation of the sidewall spalling crack. As demonstrated by McDonald (2019), based on analytical work by Ashby \& Hallam (1986), confinement increases the stress required to propagate extensional cracks and results in an effective maximum crack length (Hoek 1965; Hoek \& Martin 2014). Suppressing the propagation of the sidewall spalling crack using confinement will thus reduce the slenderness ratio, increasing the buckling stress and reducing the induced maximum compression and tension in the beam.

\subsection{Effect of non-linear elasticity (i.e. rock damage)}

McDonald (2019) demonstrated that the typical linear-elastic assumption, referring to low stress crack closure non-linearity, rather than plasticity, affects the propagation of extensional cracks. The common methodology of measuring the tangent Young's modulus at $50 \%$ of the peak load, and assuming that this is representative of the rock under the full range of stress conditions, is not valid for brittle crack propagation problems. A brief summary of McDonald (2019) is necessary to justify the methodology used in this paper.

The tensile stress at a crack tip can be calculated using analytical solutions. These solutions are derived using the assumption that the Young's modulus is constant and equal in both compression and extension. While this assumption is not correct in cracked solids, of which rocks are a classic example, there has been no compelling reason to implement the observed non-linearity. This was a reasonable justification given the difficulty involved in the implementation of non-linear deformation. The tensile stress at the tip of a sliding crack is proportional to the slip on the crack, and the Young's modulus at the crack tip. The slip length is simultaneously inversely proportional to the Young's modulus surrounding the crack. Analytical solutions (Basista \& Gross 1998) assume that these terms cancel each other. While the rock close to the crack tip is in extension, with deformation proportional to the extensional Young's modulus, the rock surrounding the sliding flaw is relatively confined, with deformation proportional to the Young's modulus in compression. The tensile stress at the crack tip is therefore affected by the ratio of these terms, defined as the bimodular ratio (see Equation 2). 


$$
B M=\frac{E_{\text {extension }}}{E_{\text {compression }}}
$$

where:

$$
\begin{array}{ll}
B M & =\text { Bimodular ratio. } \\
E_{\text {extension }} & =\text { Young's modulus in extension. } \\
E_{\text {compression }} & =\text { Young's modulus in compression. }
\end{array}
$$

In order to incorporate recoverable non-linear elasticity, the constitutive model must vary depending on the magnitude and orientation of the loading conditions. When stresses are neither hydrostatic nor above the crack closure stress in every orientation, crack closure creates anisotropy that depends on the magnitude and orientation of principal stresses. Stresses vary in both time and space during modelling of fracture growth, requiring that each zone in the model have a unique elasticity defined by the constitutive model that continuously updates as stresses in the model change.

Incorporating non-linearity in the continuum surrounding the propagating sidewall crack reduces the tensile stress at the crack tip, increases the stress required to initiate cracking, and increases the stress required to propagate the crack to a given length. These effects reduce the slenderness ratio of the beam for a given boundary condition.

Considering the tensile stress caused by flexure of the beam, for a constant slenderness ratio, the tensile stress is caused by extensional strain. The magnitude of the strain is independent of the response to the strain. If the extensional volume can soften in the extensional direction, simulating the opening of pre-existing cracks, the tensile response to a finite extensional strain will be reduced. The potential for snap-though beam failure will be reduced as the degree of non-linearity in the beam is increased. This is a possible explanation for why only some rock masses are susceptible to brittle buckling failures. This is also a potential source of leverage for reducing the potential for this type of failure. Creating damage in the rock mass by engineering small-scale cracks can increase the non-linearity in the beams and reduce the potential for buckling. These effects are investigated using numerical models, described in the following sections.

\section{Crack propagation numerical model}

Two cases are assessed:

\section{Linear rock mass.}

2. Non-linear rock mass.

The model is constructed using the methodology described by McDonald (2019), using FLAC ${ }^{3 D}$ (Itasca Consulting Group Inc. 2012). An initial isolated flaw is located in the sidewall of a 2D drift. The potential wing crack is initially intact and can propagate incrementally. Crack dimensions and properties are summarised in Table 1. Elastic properties of the rock mass are summarised in Table 2. The wing crack friction angle, cohesion, shear stiffness, and normal stiffness are set to very high values to approximate an attached interface.

Table 1 Interface properties for the initial sliding flaw and the propagating wing crack

\begin{tabular}{lcccccc}
\hline & $\begin{array}{c}\text { Normal } \\
\text { stiffness } \\
(\mathrm{GPa} / \mathrm{m})\end{array}$ & $\begin{array}{c}\text { Shear } \\
\text { stiffness } \\
(\mathrm{GPa} / \mathrm{m})\end{array}$ & $\begin{array}{c}\text { Friction } \\
\text { angle } \\
\left({ }^{\circ}\right)\end{array}$ & $\begin{array}{c}\text { Cohesion } \\
(\mathrm{MPa})\end{array}$ & $\begin{array}{c}\text { Tension } \\
(\mathrm{MPa})\end{array}$ & $\begin{array}{c}\text { Residual } \\
\text { tension } \\
(\mathrm{MPa})\end{array}$ \\
\hline Initial flaw & 10 & 10 & 0 & 0 & 0 & 0 \\
Wing crack & - & - & - & - & 10 & 0 \\
\hline
\end{tabular}


Table 2 Rock mass elastic properties

\begin{tabular}{lccc}
\hline Rock mass & $\begin{array}{c}\text { Young's modulus in } \\
\text { compression (GPa) }\end{array}$ & $\begin{array}{c}\text { Young's modulus in } \\
\text { extension (GPa) }\end{array}$ & $\begin{array}{c}\text { Poisson's } \\
\text { ratio }\end{array}$ \\
\hline Linear elastic & 10 & 10 & 0.2 \\
Non-linear elastic & 10 & 1 & 0.2 \\
\hline
\end{tabular}

A loading sequence is used to assess the incremental growth of the sidewall crack shown in Figure 1. An initial hydrostatic stress of $5 \mathrm{MPa}$ is initialised in the model. The tunnel is excavated and stepped to equilibrium. The crack is able to propagate during this stage. The vertical boundary stress is then incrementally increased in stages. During each stage, the vertical stress is applied to the boundaries of the model, creating unbalanced forces, and the model is stepped to equilibrium. During each stage, the sidewall stress will increase, and the spalling crack is able to propagate. The geometry of the beam, stresses applied the boundary of the beam, and the deflection of the beam are extracted from each case at each stage.

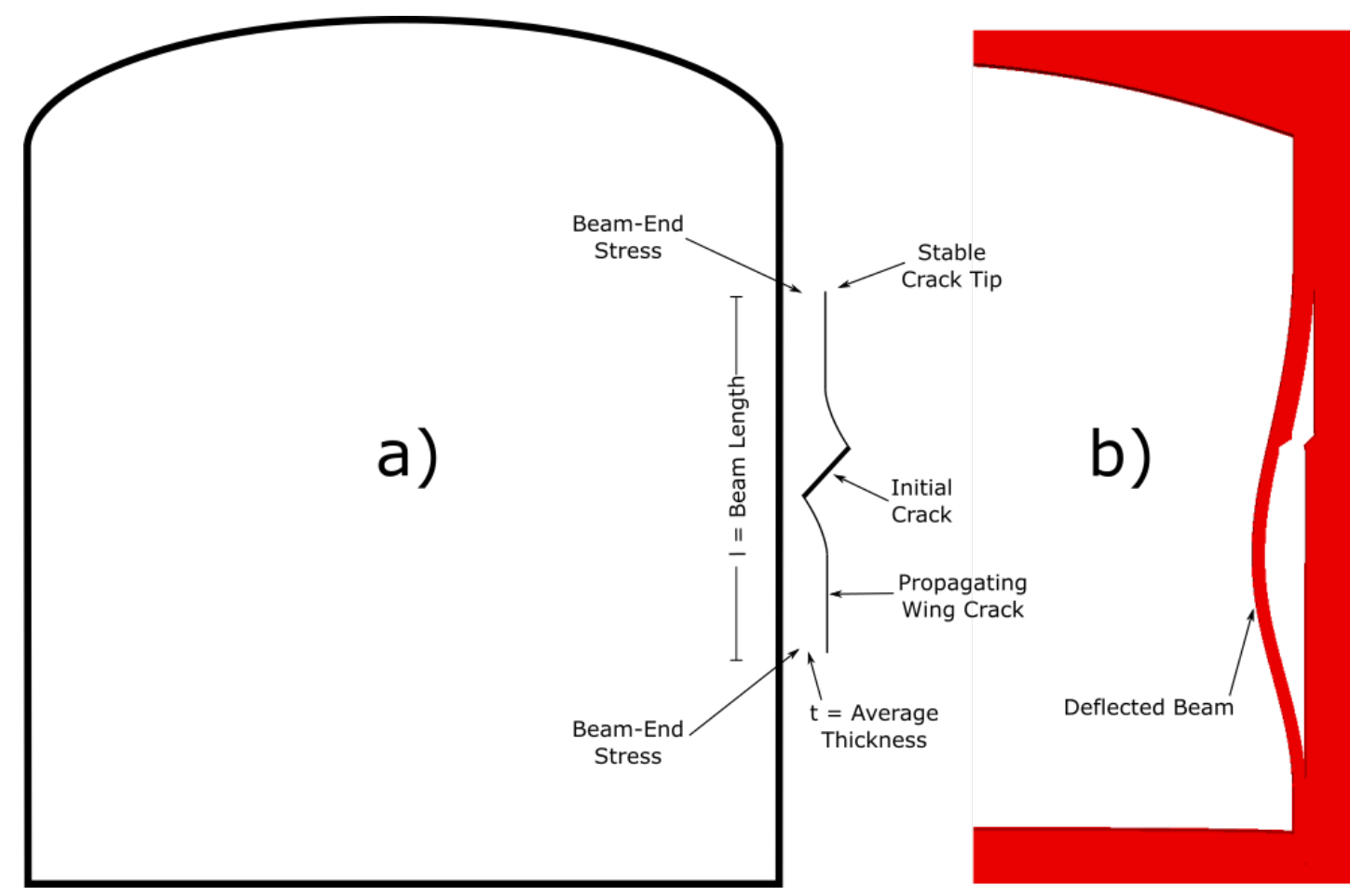

$\begin{array}{ll}\text { Figure } 1 & \text { (a) Drift geometry with an initial flaw and propagating wing crack in the sidewall; (b) Modelled }\end{array}$ deflection of a slender beam formed by propagation of the wing crack

\section{$4 \quad$ Results}

The results are analysed using two methodologies. These include an evaluation of the potential for elastic buckling and an assessment of the stresses and deflection within the beam as the boundary stress is increased.

\subsection{Elastic buckling}

For each scenario, the beam end stress and slenderness ratio are extracted at each stage. The critical Euler buckling stress (Fairhurst \& Cook 1966) is calculated based on the intact Young's modulus of the rock and compared to the model results in Figure 2. The beam-end stress is measured by the average vertical stress at the current ends of the beam, defined by the location of the wing crack tip at each stage (Figure 1). 


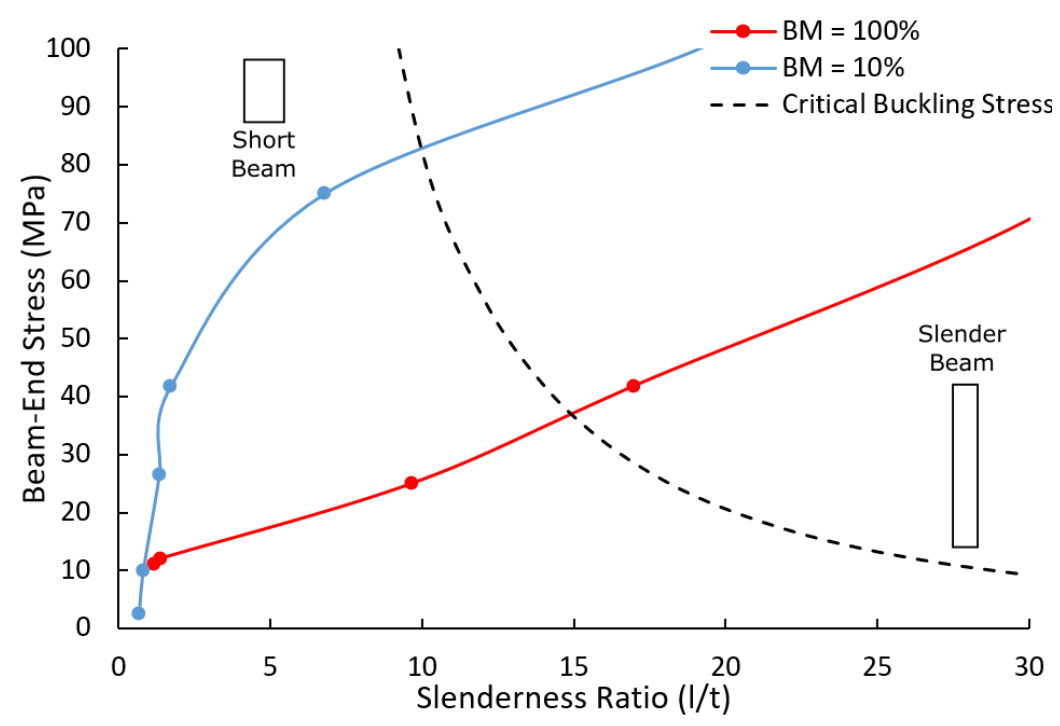

Figure 2 Evaluation of the potential for elastic buckling

\subsection{Deflection of the beam}

Deflection occurs in the beam because the loading is not perfectly aligned with the long axis of the beam, the model is not as continuous as the analytical buckling equations, and movement on the initial crack facilitates preferential deflection of the beam towards the opening. The maximum deflection of the beam is measured at each stage of the model and shown in Figure 3. The beam is consistently longer below the initial cracks than above, due to the larger beam thickness above the initial flaw (Figure 1). The maximum deflection is therefore consistently located below the initial crack.

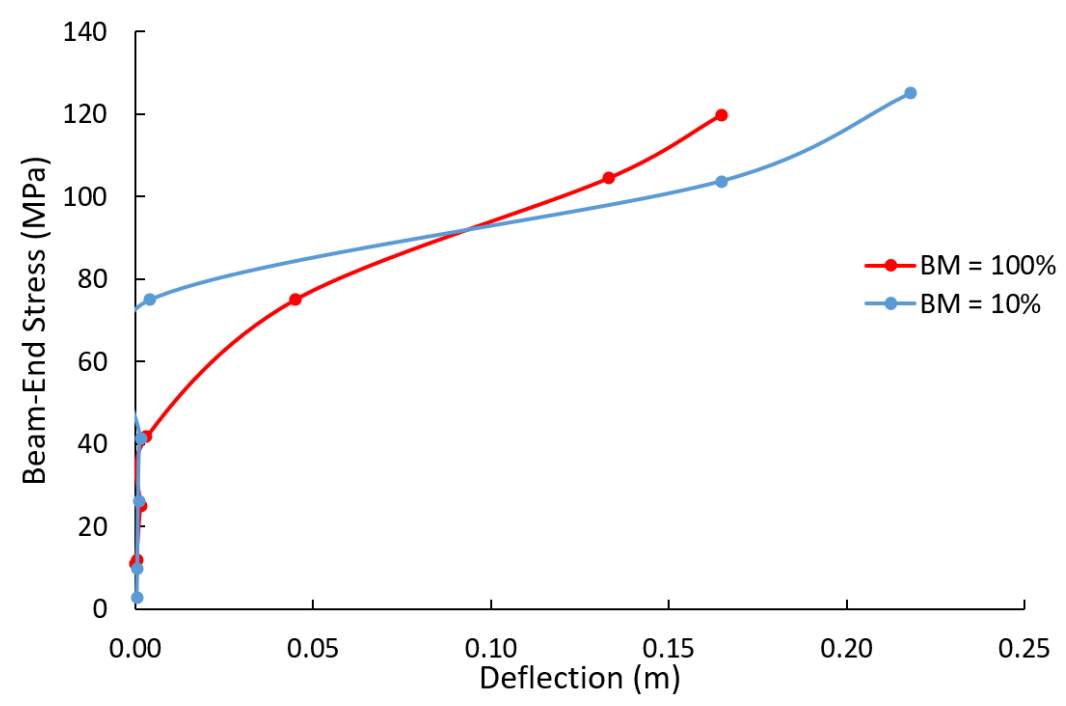

Figure 3 Evaluation of the potential for elastic buckling

\subsection{Beam stresses}

For each stage the maximum and minimum stresses are recorded in the beam. The maximum stress increases as the boundary stress increases, while the minimum stress increases initially, and then drops into tension due to deflection of the beam. The shear stress at the tip of the sliding crack increases as the boundary stresses increase, with consistently higher shear stress in the non-linear case. Beam stresses are summarised in Figure 4. 


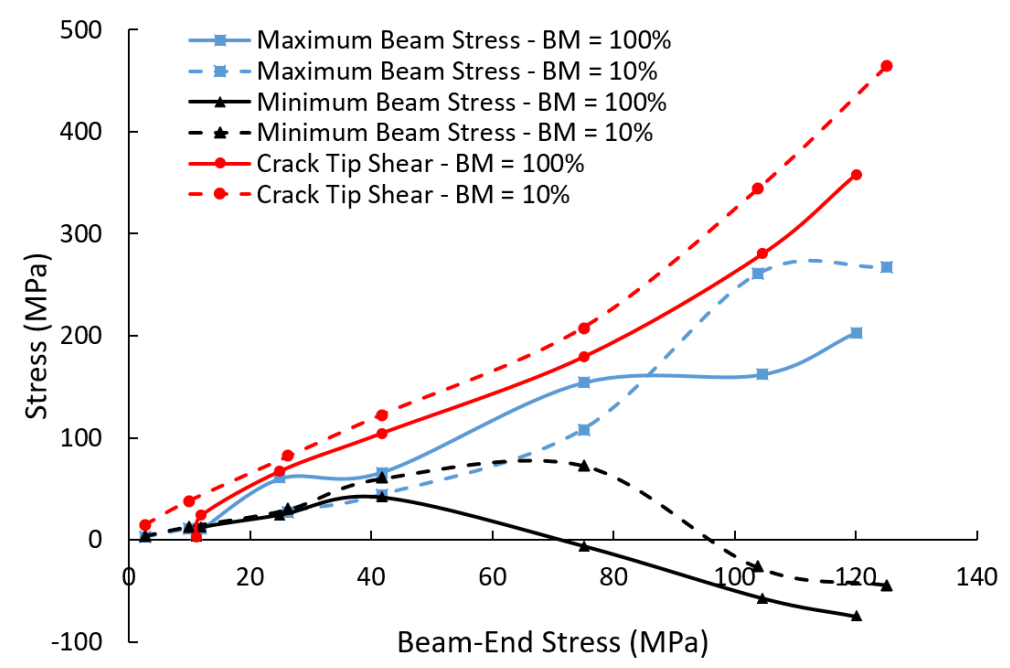

Figure 4 Maximum and minimum stress in the beam and shear stress at the sliding crack tip as the beam stress increases

\section{Discussion}

\subsection{Buckling - shear transition}

In Figure 2, the beam-end stress required to reach the critical buckling stress is $\sim 37 \mathrm{MPa}$ in the linear elastic case and $\sim 83 \mathrm{MPa}$ in the non-linear elastic case with a bimodular ratio of $10 \%$. Higher non-linearity, or damage, leads to a significant increase in stress required to trigger brittle buckling.

At the same time, the shear stress at the crack tip is increased by non-linearity. In a damaged material there are also more opportunities for crack propagation, initiating at the tips of natural and engineered flaws. When the most critical propagating cracks are suppressed, less critical cracks will initiate and propagate to a short length. This facilitates the accumulation of damage resulting in shear failure. Combined, the increase in stress required to trigger brittle buckling and the decrease in stress required to trigger less brittle shear failure promotes a transition to a less brittle mode of failure.

Shear yielding requires a relatively soft loading system for unstable failure, and consumes energy during yielding, reducing the energy available for release. Research by McDonald (2019) demonstrated that non-linearity of the continuum surrounding a shear crack reduced the brittleness of this failure mode by increasing dilation.

\subsection{Reduction of spalling through non-linearity}

The understanding of the role of non-linear elasticity in tensile crack propagation improves the recognition of high and low spalling potential. This also suggests that creating non-linearity, either through blasting damage, preconditioning techniques such as de-stress blasting, or excavation sequences that damage selected volumes of rock can reduce the likelihood for spalling in a previously higher likelihood rock mass. This interpretation is directly based on the mechanics of crack propagation. An implication is that de-stress blasting does not need to induce macro fractures to soften the rock mass. Microcrack damage, introducing non-linearity in deformation at the rock fabric level, can also reduce the likelihood of spalling. This hypothesis remains to be demonstrated through in situ de-stress blasting trials and measurement of the elastic properties of damaged and undamaged rock.

Engineering of risk mitigation strategies are currently implemented using experience, rules of thumb, and simple assumptions. While these can provide a reasonable starting point, the development of practical and effective strategies using empirical methods requires high quality well documented case histories and measurement data. These cases are used to develop techniques for identifying conditions in the field that have high likelihood of brittle failure. In the case of brittle rock mass failure, it is challenging to characterise 
rock mass conditions in advance as failures are localised and the location of a significant event is generally not forecastable. In the absence of this type of information, more rigorous mechanical assessment and improved understanding of mechanics can guide risk identification (e.g. specific combinations of geological factors and stresses) and risk mitigation strategies.

\section{Conclusion}

Spalling can be suppressed by the accumulation of damage (whether engineered or naturally occurring) to the rock mass. Rock masses that are more damaged result in shorter crack propagation, and a lower likelihood of spalling. By engineering damage into the rock mass, through such means as de-stress blasting, for example, a brittle rock mass can be made to behave in a more ductile manner. This understanding allows for the design of preconditioning strategies to directly target the rock mass rather than general ideas of reducing stress or managing the stress path on an excavation. Through a numerical model of a brittle rock mass, we show that engineering damage into the rock mass supresses and can theoretically prevent the occurrence of spalling around an underground excavation.

\section{$7 \quad$ Limitations and assumptions}

The buckling curve in Figure 3 assumes jointed end boundary conditions. This assumes that the flexure at the ends of the beams causes the ends of the beam to fracture to the excavation boundary. Laboratory testing from Handin et al. (1972) indicates that a fixed end boundary condition is more appropriate for rock beams. The critical buckling stress equation used for analysis assumes a linear-elastic material. The non-linear behaviour of rock could influence the buckling equation, but this was not considered in this investigation.

The crack was assumed to propagate on the pre-determined path defined by the explicit wing crack and the tensile strength loss on the wing crack was assumed to be brittle. Stresses at the crack tip were not perfectly aligned with this crack propagation, suggesting that the pre-determined path was not the most critical path. The extensional stress tended to rotate the critical crack to propagate towards the excavation boundary.

The constitutive model of each zone is updated every 100 timesteps because updating the constitutive model for every time step would requires significant model cycle time for each stage. Updating more frequently could improve the accuracy of the model but must be balanced against the need to reasonable calculation efficiency.

Future work can expand the range of conditions assessed (stress orientations, crack locations, crack properties, etc.), investigate the effect of crack propagation that is not limited to a pre-determined path, and investigate the effect of less brittle tensile strength loss on the wing crack.

\section{Acknowledgement}

This research was supported by Vale Canada Ltd with funding from Mitacs Canada.

\section{References}

Ashby, M \& Hallam, S 1986, 'The failure of brittle solids containing small cracks under compressive stress states', Acta Metallurgica, pp. 497-510.

Basista, M \& Gross, D 1998, 'The sliding crack model of brittle deformation: an internal variable approach', International Journal of Solids and Structures, pp. 487-509.

Bieniawski, ZT 1998, 'The Rock Mass Rating (RMR) System (geomechanics classification) in engineering practice', in L Kirkaldie (ed.), Rock Classification Systems for Engineering Purposes, ASTM International, West Conshohocken, pp. 17-34.

Fairhurst, C \& Cook, N 1966, 'The phenomenon of rock splitting parallel to the direction of maximum compression in the neighborhood of a surface', Proceedings of the First Congress on the International Society of Rock Mechanics, International Society of Rock Mechanics, Lisbon, pp. 687-692.

Handin, J, Friedman, M, Logan, J, Pattison, L \& Swolfs, H 1972, 'Experimental folding of rocks under confining pressure: buckling of single-layer rock beams', Flow and Fracture of Rocks, pp. 1-28.

Hoek, E 1965, Rock Fracture Under Static Stress Conditions, National Mechanical Engineering Research Institute Council for Scientific and Industrial Research, Pretoria. 
Hoek, E \& Martin, C 2014, 'Fracture initiation and propagation in intact rock - a review', Journal of Rock Mechanics and Geotechnical Engineering, pp. 287-300.

Hoek, E, Carranza-Torres, C \& Corkum, B 2002, 'Hoek-Brown failure criterion - 2002 edition', Proceedings of the Fifth North American Rock Mechanics Symposium and the 17th Tunnelling Association of Canada Conference, pp. 267-273. Itasca Consulting Group Inc. 2012, FLAC3D Version 5.0: User's Guide, Minneapolis.

McDonald, AC 2019, Geological Controls on Strainburst Risk in Deep Mining Drifts, PhD thesis, in review, Queen's University, Kingston. Norwegian Geotechnical Institute (NGI) 2015, Using the Q System: Rock Mass Classification and Support Design, NGI, Oslo, 56 p. 